\title{
Interactions between Species and Environments From Incomplete Information
}

\author{
Takayuki Niizato ${ }^{1}, \quad$ Yukio-Pegio Gunji ${ }^{1,2}$ \\ ${ }^{1}$ Graduate School of Science, Kobe University, Japan \\ ${ }^{2}$ Faculty of Science, Kobe University, Japan
}

e-mail: _t_niizato@yahoo.co.jp

\begin{abstract}
There are two contradictory aspects of the adaptive process in evolution. The first is that species must optimally increase their own fitness in a given environment. The second is that species must maintain their variation to be ready to respond to changing environments. In a strict sense, these two aspects might consider to be mutually exclusive. If species are optimally adapted, then the variation in the species that is suboptimal decreases and vice versa. To resolve this dilemma, species must find a balance between optimal adaptation and robust adaptation. Finding the balance between these processes requires both the local and global complete, static information. However, the balance between the processes must be dynamic. In this study, we propose a model that illustrates dynamic negotiation between the global and local information using lattice theory. The dynamic negotiation between these two levels results in an overestimate of fitness for each species. The overestimation of fitness in our model represents the multiplicity of fitness which is sometimes discussed as the exaptation. We show that species in our model demonstrate the power law of the lifespan distribution and $1 / f$ fluctuation for the adaptive process. Our model allows for a balance between optimal adaptation and robust adaptation without any arbitrary parameters.
\end{abstract}




\section{Introduction}

Adaptation is one of the intriguing issues for vast biological events such as evolution and ecology [1, 2]. Each species must survive in varied environments. Generally, we consider fitness as explaining how species adapt to an environment. "Survival of the fittest", proposed by Darwin [2], is the most famous theory for adaptation and evolution. Many models for evolution have therefore applied the concept of fitness for better or worse $[1,3,4,5]$. The celebrated model of Bak and Sneppen explained biological evolution using a fitness landscape [3, 4]. Their model is very simply constructed. Each species has its own fitness that is independent of other species, and species connect to each other in a flood chain. A selected less fit species changes its fitness randomly. The neighboring species in the food chain then also changes its value of fitness at random. Bak and Sneppen explained the biological evolution of an ecosystem; for example, the power laws for extinction's distribution, using this simple model [3, 4].

The evolutional model of Bak and Sneppen provides many suggestions for considering a species' adaptation in the fitness landscape. The problem with their model, which we address here, is the assumption of perfect information on fitness because the model must know all of the fitness values to decide the least fit. The requirement for knowing all of the precise fitness values assumption seems unnatural. From Darwin's evolutional theory, we surely see the results of a species' fitness. However, we often observe that nature permits unnecessary traits in species during their evolution. The concept of exaptation, proposed by Gould [6, 7], could explain the existence of these traits, which seem to be unnecessary. Exaptation has two meanings [7]. One is "a feature, now useful to an organism, that did not arise as an adaptation for its present role, but was subsequently co-opted for its current function". The other is a "feature that now enhance fitness, but were not built by natural selection for their current role". A famous example of exaptation is the wings of birds. It is believed that feathers were originally used for thermal regulation before they were used for flight. This example suggests that the traits of animals would potentially have various possible functions that could be adapted to many situations. Therefore, we believe that exaptation would permit multiplicity of the fitness in nature because of the way that trait usage is open in an environment. Fitness, which is imposed by the environment, is not determined definitively as in the model of Bak and Sneppen. In the living world, it is very hard to obtain precise information, such as absolutely knowing all fitness levels.

The assumption of complete precise information of fitness would be a risk for erasing the essential problem of adapting species to their environment. One typical problem is finding the balance between optimal adaptation and robust adaptation $[8,9]$. The robust adaptation means that the system must keep high diversity of species to avoid whole extinction. The optimal adaptation means that the system must contain as many species with the highest fitness as possible to increase their whole fitness. These two adaptive processes, however, are mutually exclusive. If we start from complete precise information of fitness, we do find the optimal balance between two adaptive processes because the definition of fitness is utterly imposed on the modeler $[10,11]$. If nature finds the balance between them by itself, it is important to consider what is incomplete information of fitness when we construct a model for the adaptation.

The incomplete information of fitness would be considered as the multiplicity of fitness as we discussed 
the example of exaptation. Multiplicity would provide a certain interval for the selection among species' traits when they decide the necessary-unnecessary traits for their survival. Of course, the reference point of the selection among species' traits strongly depends on the context where they live because the usage of traits also depends on their environment. On the other hand, each individual measures its fitness from its local incomplete observation to adapt to the environment. Therefore, the implementation of the multiplicity of fitness is accomplished by reflecting both aspects, which are the local conditions and the global (or their context) conditions.

In this paper we use lattice theory to represent the interface between the local and the global fitness in the adaptive process to their environment. Each species observes only parts of the environment. This incomplete local fitness is represented as fitness blocks. The fitness blocks represent the necessary-unnecessary portion in their traits by using binary bit strings. We construct a lattice from these fitness blocks. The lattice structure ensures the order relation among each fitness block and avoids the existence of non-comparable fitness block (element) with all other elements. The lattice structure also evolves with time steps because the relation among fitness blocks dynamically changes. In our evolving lattice model, local incomplete information drives the evolution of the constructed lattice. The global context of the fitness in our model represents as the congruence of the lattice. To use the congruence, the system re-estimates the incomplete local fitness using a quotient lattice. The quotient lattice plays a role in identifying a set of fitness blocks as representative one fitness blocks. We show that species in our model can obtain robust adaptation without any parameter tuning using a quotient lattice.

\section{Basic Concepts}

To construct our model, we use a lattice theory. The lattice theory has widely used in the computer science such as an automaton theory $[12,13]$. Here we review the basic definitions and notion, which are used in our model for unfamiliar readers of the lattice theory.

Definition 2.1 (Partial Order) Let $P$ be a set. An order on $P$ is a binary relation $\leq$ on $P$ such that, for all $x, y$, $z \in P$

(i) $x \leq x$

(ii) $x \leq y$ and $y \leq x \Rightarrow x=y$

(iii) $x \leq y$ and $y \leq z \Rightarrow x \leq z$

We denote a partially ordered set by the pair, $(P, \leq)$. For example, a set of bit (binary) strings can construct a partial order. A bit string $a_{1} a_{2} a_{3} \ldots a_{n}$ is a finite sequence of zero or one $\left(a_{i} \in\{0,1\}\right)$. An order between two bit strings such as $a_{1} a_{2} a_{3} \ldots a_{n}$ and $b_{1} b_{2} b_{3} \ldots b_{n}$ is defined by $a_{1} a_{2} a_{3} \ldots a_{n} \leq b_{1} b_{2} b_{3} \ldots b_{n}$ if $a_{i} \leq b_{i}$ for all $i$. We use a set of bit strings in this study. However, A partial order is not a lattice. Then we define the meet and the join. We define the join " $\vee$ ” and the meet “ $\wedge$ ” of two elements $x$ and $y$ in $P$. The join can be defined by $x \vee y$ 
$=\sup \{x, y\}$ when it exists. The join can be defined by $x \wedge y=\inf \{x, y\}$ when it exists. The notation of sup (inf) means the lowest (greatest) upper bound of $\{x, y\}$ in $P$.

Definition 2.2 (Lattice) Let $(P, \leq)$ be a non-empty partially ordered set.

If $x \vee y$ and $x \wedge y$ exist for all $x, y \in P$, then $(P, \leq)$ is called for a lattice.

To distinct a partially ordered set, we denote a lattice as $(L, \leq, \wedge, \vee)$. In this paper, there are often-used sets that are an ideal and a filter. An ideal is used when we construct the congruence on a lattice.

Definition 2.3 (Ideal) Let $(L, \leq, \wedge, \vee)$ be a lattice. A non-empty subset of $J$ is called an ideal if

(i) $x, y \in J$ implies $x \vee y \in J$,

(ii) $x \in L, y \in J$ and $x \leq y$ imply $x \in J$.

Definition 2.4 (Filter) Let $(L, \leq, \wedge, \vee)$ be a lattice. A non-empty subset of $F$ is called an filter if

(i) $x, y \in F$ implies $x \wedge y \in F$,

(ii) $x \in L, y \in F$ and $y \leq x$ imply $x \in F$.

The typical example of an ideal is a down set on a lattice. The definition of a down set is a subset $J=\{y \in L \mid$ $y \leq x\}$ when $x \in L$. We denote a down set of $x$ as $\downarrow_{x}$. It can easily be rerified that a down set satisfies the condition of ideal. In a similar way, we can define an upper set on a lattice such as $F=\{y \in L \mid x \leq y\}$ when $x$ $\in L$. We denote an upper set of $x$ as $\uparrow x$. We can also rerify that an upper set satisfies the condition of a filter. Next, we consider congruence on a lattice to define a quotient lattice. A congruence is an equivalence relation which is restricted by a certain condition.

Definition 2.5 (Congruence on a Lattice) Let $(L, \leq, \wedge, \vee)$ be a lattice. Let an equivalence relation on $L$ be $\theta=$ $\{<x, y>\in L \times L\}$ such that any $x, y, z \in L$,

(i) $<x, x>\in \theta$

(ii) $<x, y>\in \theta \Leftrightarrow<y, x>\in \theta$

(iii) $<x, y>\in \theta$ and $<y, z>\in \theta \Rightarrow<x, z>\in \theta$

We also denote $<x, y>\in \theta$ as $x \equiv y(\bmod \theta)$. Then an equivalence relation is a congruence on $L$, if for any $x, y$, $z, w \in L, \quad(x \equiv y(\bmod \theta)$ and $z \equiv w(\bmod \theta)) \Rightarrow(x \vee z \equiv y \vee w(\bmod \theta)$ and $x \wedge z \equiv y \wedge w(\bmod \theta))$

Then we can make a quotient lattice by using a congruence.

Definition 2.6 (Quotient Lattice) Let $\theta$ be a congruence on a lattice $(L, \leq, \wedge, \vee)$, then a set $L / \theta$ is defined by 
$L / \theta=\left\{[x]_{\theta} \mid x \in L\right\}$ with $[x]_{\theta}=\{y \in L \mid x \equiv y(\bmod \theta)\}$

The join and the meet on $L / \theta$ are defined by

$$
[x]_{\theta} \wedge[y]_{\theta}:=[x \wedge y]_{\theta},[x]_{\theta} \vee[y]_{\theta}:=[x \vee y]_{\theta}
$$

Then we call $(L / \theta, \leq, \wedge, \vee)$ the quotient lattice of $L$ modulo $\theta$.

In this study, we construct a quotient lattice from a given ideal. First we introduce the equivalence relation derived from an ideal.

Definition 2.7 (Equivalence Relation Derived from an Ideal) Let $J$ be an ideal on a lattice $L$. The equivalence relation derived from an ideal $J$ is;

$$
\theta(J):=\{\langle x, y>\in L \times L| \exists z \in J x \vee z=y \vee z\}
$$

We can easily rerify that Definition 2.7 satisfies Definition 2.5 and that $\theta(J)$ is well-defined to be a congruence relation. Then next proposition ensures the ideal as a block of the partition derived by a quotient lattice. This proposition would be used when we discuss the existence of the lowest block information.

Proposition 2.8 Let $\theta(J)$ be an equivalence relation derived from an ideal $J$ on a lattice $L$. Then $J$ is a block of the corresponding partition of $L$.

Proof. $J$ is a block of the corresponding partition of $L$ iff $\forall x \in J,[x]_{\theta(J)}=J$

Therefore we must prove $(*)$.

(i) $\forall y \in[x]_{\theta(J)} \Leftrightarrow(\exists z \in \theta(J)) x \vee z=y \vee z \Rightarrow x \vee Z=y \vee z \in J$ (Definition 2.3) $\Rightarrow y \leq y \vee z, y \in J$ (Definition 2.3)

(ii) Supposing $y \in J$, for $[x]_{\theta(J)}$, it is trivial that $x \in J$, then $x \vee y \in J$. Clearly, $x \vee(x \vee y)=y \vee(x \vee y)$. It entails $y$ $\in[x]_{\theta(J)}$

From (i) and (ii), we proved $[x]_{\theta(J)}=J$.

We also point out that a quotient lattice is deeply connected to a map between lattices, which is called a homomorphism.

Definition 2.9 (Homomorphism) Let $L$ and $K$ be a lattices. A map $f: L \rightarrow K$ is said to be a homomorphism if for any $x, y \in L$, 
(i) $f(x \wedge y)=f(x) \wedge f(y) \quad$ (meet-preserving)

(ii) $f(x \vee y)=f(x) \vee f(y) \quad$ (join-preserving)

Especially, $f$ is called isomorphism when $f$ is a bijective homomorphism.

Proposition 2.10 (Homomorphism between a Lattice and a Quotient Lattice) Let $\theta$ be a congruence on a lattice $L$. Then $(L, \leq, \wedge, \vee)$ is a lattice and a natural quotient map $f: L \rightarrow L / \theta$, defined by $f(x):=[x]_{\theta}$, is a homomorphism.

Proof. We check that a natural quotient map $f$ satisfies Definition 2.5.

$$
\begin{aligned}
& f(x \vee y)=[x \vee y]_{\theta}=[x]_{\theta} \vee[y]_{\theta}=f(x) \vee f(y) \\
& f(x \wedge y)=[x \wedge y]_{\theta}=[x]_{\theta} \wedge[y]_{\theta}=f(x) \wedge f(y)
\end{aligned}
$$

The next theorem is often used in this paper. The theorem is used for constructing a quotient lattice from an ideal. Using this theorem, we can determine a quotient lattice from a given lattice.

Theorem 2.11 (Reconstruction of a Lattice from a Quotient Lattice) Let $L$ be a lattice and $f$ be a natural quotient map such as $f: L \rightarrow L / \theta$. For the binary relation derived from an ideal $J \subseteq L$, there exists a filter $K \subseteq$ $L$ such that $[x]_{\theta(J)}=f^{-1}(x)$, where for any $x \in K, f^{-1}(x):=\downarrow_{x}-\cup_{y \in K, y<x} \downarrow y$

Proof. See [14].

\section{Model}

In our model, we represent genotypes, species and other individual property as binary bit strings. Bit strings are constructed from a sequence of zeros and ones, for example 010011. Formally, an $n$-bit string can represent $a_{1} a_{2} a_{3} \ldots a_{n}$ and $a_{i} \in\{0,1\}$ for all $i \in\{1,2, \ldots, n\}$. We briefly denote $a_{1} a_{2} a_{3} \ldots a_{n}$ as $\langle a\rangle_{n}$. In this paper we use three types of binary bit strings. One type represents a species in a given niche. A second one represents an environment (or a target). We denote $\langle t\rangle_{n}$ and $\langle s\rangle_{n}$ for a target bit string and a species bit string, respectively. We consider an ecosystem consisting of species, which are represented by binary $n$-bit strings, as a set $M$, where $M=\left\{\left\langle s^{1}\right\rangle_{n},\left\langle s^{2}\right\rangle_{n}, \ldots,\left\langle s^{m}\right\rangle_{n}\right\}$. The number of elements in the set $M$ is fixed at $m$, which indicates the niche size. Each individual attempts to adapt its target bit string $\langle t\rangle_{n}$.

However, these binary bit strings, which represent species or the environment, have no relationships between them. The order structure of the binary bit strings can be defined by the fitness, which is measured by fitness blocks. The definition of a fitness block is as follows.

Definition 3.1 (fitness Block) Let $\langle s\rangle_{n}$ and $\langle t\rangle_{n}$ be a $n$-bit strings. $\langle s\rangle_{n}$ is a species, and $\langle t\rangle_{n}$ is a target. $k$ is a hidden digit of a target bit string. 
For $1 \leq j \leq n$,

$b_{j}=\left\{\begin{array}{c}0 \text { (if } a_{j}=t_{j} \text { and } j \neq k \text { ) } \\ 1 \text { (if } a_{j} \neq t_{j} \text { and } j \neq k \text { ) } \\ 0 \text { or } 1 \text { (otherwise) }\end{array}\right.$

We call $\langle b\rangle_{n}$ the species' fitness block for a target.

Obviously, the fitness bit strings makes a partial order set (Supporting Information). A hidden digit represents the imperfect knowledge of an environment because each species never has a perfect knowledge about its environment. Each species can only observe $n-1$ bits from a target $n$-bit string. The hidden digit in a target bit string is randomly selected for each time step. If a hidden digit is $k(1 \leq k \leq n)$ at time $t$, each species can observe a target bit string $t_{1} t_{2} \ldots t_{k-1} t_{k+1} \ldots t_{n}$. From Definition 3.1, we observe that species' fitness blocks $\langle b\rangle_{n}$ of the target is also a binary $n$-bit string. An adaptation in our model means that a species tries to mimic a target bit string. In other words, we presume that a species' bit string has high fitness in a given environment when it mimics a target bit string well. Therefore, each species must leave some portions that were held in common with the target and change other portions that were difference from the target to raise its own fitness. Fitness blocks represent information on the changing and unchanging digits for each species in a given niche. We defined changing digits as zeros and unchanging digits as ones. The value of a hidden digit is determined to be zero or one randomly. A hidden digit also represents an uncertain environment because a hidden digit in the target bit string changes every step. A hidden digit, therefore, not only represents imperfect information but also an uncertain environment for each species.

In doing this, we obtain all fitness blocks for the species, which is a set $B=\left\{\left\langle b^{1}\right\rangle_{n},\left\langle b^{2}\right\rangle_{n}, \ldots,\left\langle b^{l}\right\rangle_{n}\right\}$. The number of elements in set $B$ is less than the number of elements of $M(|B| \leq|\mathrm{M}|$; we denote $|-|$ as a cardinality) because it contains binary bit strings that can be identical for some species. For example, consider 3-bit strings. A target that has no hidden digits is represented by 011, and the species are represented by $\{010,111$, $010\}$. The set of binary bit strings in the fitness block is $\{110,011\}$. We also use a set of binary bit strings for fitness blocks that contain the same fitness block (in the case of above example $\{110,011,110\}$ ). We denote this set as $\bar{B}$.

The order of binary bit strings (fitness blocks) corresponds to the fitness rank. In a set of binary bit strings, an order between two binary bit strings is defined by $\langle a\rangle_{n} \leq\langle b\rangle_{n}$ if $a_{i} \leq b_{i}$ for all $i \in\{1,2, \ldots, n\}$. Therefore, a set of $n$-bit strings in a fitness block can produce a partially order set. We also define the meet and the join in $n$-bit strings.

Definition 3.2 (Meet and Join of n-Bit String) Let $\langle a\rangle_{n}$ and $\langle b\rangle_{n}$ be a $n$-bit strings.

(meet) $\langle a\rangle_{n} \wedge\langle b\rangle_{n}:=\min \left\{a_{1}, b_{1}\right\} \min \left\{a_{2}, b_{2}\right\} \ldots \min \left\{a_{n}, b_{n}\right\}$

(join) $\langle a\rangle_{n} \vee\langle b\rangle_{n}:=\max \left\{a_{1}, b_{1}\right\} \max \left\{a_{2}, b_{2}\right\} \ldots \max \left\{a_{n}, b_{n}\right\}$

The notation of $\max S$ (minS, resp.) indicates the largest (smallest, resp.) element in a set $S$. 
A set of fitness blocks constructs a partially ordered set but does not create a lattice because some pairs of elements in this set are not covered by the join and the meet. This suggests that there are some elements, which are not comparable, in the set by the order relation. This does not fit with the concept of the fitness. Therefore we need to construct a lattice structure from a given set of binary bit strings. In this study we use a topped-intersection structure to construct the lattice.

Definition 3.3 (Topped-Intersection Structure in Bit Strings) Let $L$ be a set of $n$-bit strings. If $L$ is a topped-intersection structure, then $L$ satisfies conditions (i) and (ii).

For any $S \subseteq L$,

(i) $\wedge S \in L$

(ii) $\vee L \in L$

Definition 3.3 indicates that a topped intersection structure is closed under the meet. In other words, a topped intersection structure contains any pair of elements for the meet operation. We consider 3 bit strings again. A target, which has no hidden digit, is represented by 011, and the species are represented by $\{010$, $111,010\}$. Thus, a block information set is $\{110,011\}$. The topped intersection structure of $\{110,011\}$ is $\{010,110,011,111\}$ because 111 is the top element and $010=110 \wedge 011$. Therefore $\{010,110,011,111\}$ makes a lattice. We denote a lattice, which is constructed from fitness blocks, as L. With a topped intersection structure, we are able to obtain global information between fitness blocks about the ordered relationship.

We defined fitness blocks as required blocks for raising the fitness of each species. However, a decision regarding fitness blocks involves a contextual constraint. We can correspond this contextual constraint to a quotient lattice because the equivalence relations of a lattice are restricted by the global structure (or the relation among the fitness). Generating a quotient lattice operates at the interface between local fitness and global context. Before we discuss how to determine an ideal, we define fitness blocks being induced by a quotient lattice. The fitness block that is induced by a quotient lattice is defined as follows.

Definition 3.4 (Fitness block Being Induced by a Quotient Lattice) Let $L$ be a lattice of $n$-bit strings, $J$ be an ideal and $\theta(J)$ be a congruence on lattice $L$. For $1 \leq i \leq|B|$,

$$
\left\langle b^{i}\right\rangle_{n} \leftarrow \vee\left\{\langle x\rangle_{n} \in L \mid\left[\langle x\rangle_{n} \in\left[\langle a\rangle_{n}\right]_{\theta(J)} \text { such that }\left\langle b^{i}\right\rangle_{n} \in\left[\langle a\rangle_{n}\right]_{\theta(J)}\right]\right.
$$

We denote $\leftarrow$ as the substitution operation. Therefore, a binary bit string of fitness blocks is replaced by the largest element in the congruence to which it belongs.

A selected element corresponds to the top of the congruence to which it belongs based on Definition 2.7. 
Here we consider the meaning of congruence for the adaptive process. In our model, the binary bit string of a fitness block is replaced by the largest element in the congruence to which it belongs. From the fitness block definition, this operation fixes some digits in the binary bit string that should be able to change. In other words, each species overestimates its own fitness by introducing the congruence because a substituted element is always larger than the original element according to Definition 3.4. Why do we consider such an overestimation of the fitness? In the adaptation of a living organism, it is more important to retain traits that appear unnecessary than to discard traits that are necessary. The attitude of maintaining unnecessary traits suggests multiple measurements of the fitness (we have previously discussed such situations on exaptation and pre-adaptation [6, 7]). In our model this multiplicity would emerge as an overestimation of the fitness. The overestimation of the fitness suggests that fitness blocks for each species has a meaning beyond the local fitness because the overestimation of the fitness is generated from the negotiation between the global information (an element in the quotient lattice) and the local information (an element in a lattice). This definition of the overestimation of the fitness, or multiplicity of the fitness, matched with our discussion, which we argued in Introduction.

Of course, the overestimation of the fitness is never determined randomly but is rather constrained by the global and historical context. With a quotient lattice, we can determine the overestimation of fitness by the well-defined way mathematically. The quotient lattice corresponds to the global context because the ordered relationship of the fitness blocks (fitness) contains global information. What about the impact of historical context on the overestimation of fitness? The historical context of the overestimation would correspond to the method of determining the congruence. In other words, it corresponds to determining an ideal, which means a down set in a lattice, in our model because the selection of an ideal would affect the construction of the quotient lattice. In a finite, partially ordered set, an ideal precisely corresponds to a down set. Therefore, the selection of the ideal $\downarrow\langle x\rangle_{n}$ means the selection of an element $\langle x\rangle_{n}$ in a lattice $L$. To determine $\langle x\rangle_{n}$, we define a "reference point" in the lattice. If the reference point is determined, an ideal is determined using the following rule.

Rule 1 (Determining an Ideal) Let $L$ be a lattice and $\langle r\rangle_{n} \in L$ be a reference point. An ideal is $\downarrow\langle x\rangle_{n}$ where $\langle x\rangle_{n}$ is randomly selected from a filter, $\uparrow\langle r\rangle_{n}$.

Rule 1 suggests that the top element of an ideal is selected from the upper set (filter) of the reference point. The selection from the upper set is due to the overestimation of the fitness, which we discussed previously. The reference point is the base element in the selection of the top element of an ideal and is transferred to the next generation. This inheritance of the reference point provides a historical context to the overestimation of fitness. The rule of the succession of the reference point is as follows.

Rule 2 (Determining a Reference Point) Let $L^{t}$ be a lattice of $n$-bit strings, $\langle r\rangle_{n}{ }^{t}$ be a reference point and $\bar{B}^{t}$ be a set of fitness blocks that contains the same fitness block at time $t$. Then a reference point at time $t+1$ is 


$$
\langle r\rangle_{n}^{t+1}=\left\{\begin{array}{c}
\text { (i) }\langle r\rangle_{n}^{t} \text { (if }\langle r\rangle_{n}^{t} \in L^{t+1} \text { and }\langle r\rangle_{n}^{t} \neq\langle 0\rangle_{n}^{t} \text { ) } \\
\text { (ii) randomly selected }\langle x\rangle_{n}^{t+1} \in\left\{\langle r\rangle_{n}^{t} \wedge\langle y\rangle_{n}^{t+1}\langle y\rangle_{n}^{t+1} \in L^{t+1} \text { and }\langle r\rangle_{n}^{t} \wedge\langle y\rangle_{n}^{t+1} \in L^{t+1}\right\} \\
\text { (if }\langle r\rangle_{n}^{t} \notin L^{t+1} \text { and }\langle r\rangle_{n}^{t} \neq\langle 0\rangle_{n}^{t} \text { and }\left\{\langle r\rangle_{n}^{t} \wedge\langle y\rangle_{n}^{t+1}\langle y\rangle_{n}^{t+1} \in L^{t+1} \text { and }\langle r\rangle_{n}^{t} \wedge\langle y\rangle_{n}^{t+1} \in L^{t+1}\right\} \neq \phi \text { ) } \\
\text { (iii) randomly selected }\langle b\rangle_{n}^{t+1} \in \bar{B}^{t+1} \text { (otherwise) }
\end{array}\right.
$$

$\langle 0\rangle_{n}{ }^{t}$ indicates an all zero $n$-bit string $00 \ldots 0$ in a lattice $L$. The original reference point is randomly selected from a set $\bar{B}^{t}$ (Rule 2(iii)). The reference point reflects the proportion of the population from the fitness block. The inheritance of the reference point to next generation corresponds to Rule 2 (i) and 2 (ii). Rule 2 (i) means that the reference point is passed on to the next lattice $L^{t+1}$ if it exists in the next lattice $L^{t+1}$. However, if the reference point does not exist in lattice $L^{t+1}$, we take an element from a non-empty set of all meets with in the lattice $L^{t+1}$. If the reference point is an all zero bit string or a set of all meets and the next lattice is empty, the reference point is again selected randomly from the set $\bar{B}^{t}$. The reference point must always reflect the proportion of the fitness block. Therefore, the reference point must be checked as to whether it reflects the proportion of the fitness block or not because the origin of the reference point is set $\bar{B}^{t}$. This checking procedure leads to Rule 3.

Rule 3 (Checking a Reference Point) Let $L^{t}$ be a lattice of $n$-bit strings, $\langle r\rangle_{n}{ }^{t}$ be a reference point and $\bar{B}^{t}$ be a set of fitness blocks that contain the same fitness blocks at time $t$. Then next rule for the reference point when $\langle b\rangle_{n}{ }^{t}$ is randomly selected from $\bar{B}^{t}$ is as follows.

(i) Use Rule 2 for the next step if $\left(\langle b\rangle_{n}^{t} \in \uparrow\langle r\rangle_{n}^{t}\right)$

(ii) otherwise, randomly selected $\langle b\rangle_{n}^{t+1} \in \bar{B}^{t+1}$ for next step

Rule 3 applies to the selection of the reference point for next step. To check the validity of the reference point, we use the filter (upper set) of the reference point. This filter corresponds to possible elements of the overestimation of the fitness. Rule 3 requires taking a new reference point from set $\bar{B}^{t+1}$ for next step if the reference point does not reflect the proportion of fitness block.

So far, we have considered the global and the historical property of overestimating fitness with a reference point. A quotient lattice induced by the reference point plays an important role in our evolving lattice model. The overestimation of the fitness would generally give us an impression of the error of measurement. In 
other words, the determined fitness block is not an optimal strategy for species at the time. We shall show later that this errant fitness block contributes to generating a diversification in species. We also point out here that errors induced by the overestimation of the fitness are not described by stochastic properties. The difference between them will be argued in the next section.

Finally, we summarize the algorithm of our model below.

\section{Algorithm}

(1) Generate $m n$-bit strings $\left\langle s^{1}\right\rangle_{n}{ }^{0},\left\langle s^{2}\right\rangle_{n}{ }^{0}, \ldots\left\langle s^{m}\right\rangle_{n}{ }^{0}$ and a target $n$-bit string $\langle t\rangle_{n}$ randomly.

(2) Randomly select a hidden digit of the target from set $\{1,2, \ldots, n\}$. An fitness block is formed from a set of $n$-bit strings $M^{t}=\left\{\left\langle s^{1}\right\rangle_{n}{ }^{t},\left\langle s^{2}\right\rangle_{n}{ }^{t}, \ldots,\left\langle s^{m}\right\rangle_{n}{ }^{t}\right\}$ using Definition 3.1. A set of binary bit strings for fitness blocks is denoted $B^{t}=\left\{\left\langle b^{1}\right\rangle_{n}^{t},\left\langle b^{2}\right\rangle_{n}^{t}, \ldots,\left\langle b^{t}\right\rangle_{n}{ }^{t}\right\}$.

(3) Make a lattice $L^{t}$ with a topped intersection structure of $B^{t}$ (Definition 3.3).

(4) Determine a reference point $\langle r\rangle_{n}{ }^{t}$ in a lattice $L^{t}$ using Rule 2 and check the reference point using Rule 3.

(5) Make an ideal $\downarrow\langle x\rangle_{n}{ }^{t}$ from the reference point $\langle r\rangle_{n}{ }^{t}$ using Rule 1. Construct a quotient lattice from the ideal $\downarrow\langle x\rangle_{n}{ }^{t}$.

(6) Substitute new binary bit strings of fitness blocks using Definition 3.4.

(7) Change each $n$-bit string using fitness blocks. For $1 \leq j \leq n$ and $1 \leq i \leq m$,

$$
s_{j}^{i, t+1}=\left\{\begin{array}{c}
\left.s_{j}^{i, t} \quad \text { if } b_{j}^{i, t}=1\right) \\
0 \text { or } 1 \text { (otherwise) }
\end{array}\right.
$$

Construct a new set of binary bit strings for species $M^{t+1}=\left\{\left\langle s^{1}\right\rangle_{n}{ }^{t+1},\left\langle s^{2}\right\rangle_{n}{ }^{t+1}, \ldots,\left\langle s^{m}\right\rangle_{n}{ }^{t+1}\right\}$.

We define steps as (2)-(7) as one time step. Repeating steps (2)-(7), we can observe the evolution of the species and the lattice. Figure 1a is an example of an evolving lattice using our algorithm. The procedure for the algorithm is listed on each panel in Figure 1a. In our model, each species forms a fitness block. This fitness block makes a lattice and generates new fitness block using a reference point. Finally, the substituted fitness block changes the state of species. All patterns for the selection of a reference point are listed in Figure 1b and 1c. Figure 1b corresponds to Rule 2 (i) and Figure 1c corresponds to Rule 2 (ii). Rule 2 (iii) is described in Figure 1a. In this way species in our model make a lattice from local imperfect information (fitness blocks) and re-estimate their fitness from the constructed quotient lattice, which is induced by the 
reference point.

\section{Results}

We set all possible configurations for an $n$-bit string. The total number of $n$-bit strings is $2^{n}$. For 2-bit strings, a set of all possible configurations is $\{00,01,10,11\}$. This number of settings means that one $n$-bit string in all kinds of $n$-bit strings has potentially one niche. We tried other cases by changing the number of bit strings. However, there no essential difference between them was found. Therefore, we fix the number of individuals at $2^{n}$. There is no additional parameter to our model.

We examine a time series of the evolving lattice model. In this simulation, we consider a set of 8-bit strings. Figure 2a is the time series for the number of elements in the evolving lattice, which is given as $\left|L^{t}\right|$ ( $|S|$ denotes the number of elements in set $S$ ). The number of elements in a lattice can be observed to dynamically change at every step. This finding suggests that the global structure, which results from the local fitness, is not static. Therefore, the relationship between the globality and locality of the model keeps changing. Figure $2 \mathrm{~b}$ shows the time series of the averaged distance to the target bit string. The averaged distance, $d$, can be computed with the following mathematical expression.

$$
d^{t}=\frac{1}{m} \sum_{i=1}^{m} \sum_{j=1}^{n} \frac{\left|t_{j}-s_{j}^{i, t}\right|}{n}
$$

We point out that the average distance also contains information on the fitness of the entire species. A bit string has the highest fitness when it is consistent with the target bit string. The average distance, therefore, is the degree of fitness of the entire species. Figure $2 \mathrm{~b}$ shows that the average distance oscillates around $d=1.1$. Although $d$ is large for early intervals, the value soon oscillates around a low $d$. This oscillation persists for number of time steps. The graph shows that each species attempts to mimic the target (i.e., adapt), but that many species fail to mimic their target. Therefore, the process of adaptation to the target does not reach equilibrium.

Next we discuss Figure 2c. The vertical line in Figure 2c represents the bit flip rate per bit. It can be expressed using the following mathematical expression.

$$
B F^{t}=\frac{1}{m} \sum_{i=1}^{m} \sum_{j=1}^{n} \frac{\left|s_{j}^{i, t+1}-s_{j}^{i, t}\right|}{n}
$$

The time series of bit flip rates appears to be white noise (indeed, the slope of the power spectrum is -0.22 ). The bit flip rate does not remain positive; it sometimes becomes zero, which indicates that no bit flip event occurred over the interval. A lack of a bit flip event can occur when an ideal fully covers a lattice. All fitness blocks change to $111 . . .11$ according to Definition 3.4 when an ideal covers an entire lattice (mathematically 
when $\left|L^{t} / \theta(J)\right|=1$ ). The degree of the bit flip rate roughly depends on the structure of the quotient lattice, constructed by fitness blocks because of the way the selected ideal $J$ affects the changing or failing to change digits for each bit string. Generally, the bit flip rate becomes larger when the number of elements in a quotient lattice (mathematically $\left|L^{t} / \theta(J)\right|$ ) increase. Figure 2d shows the correlation between the bit flip rate and the log transformation of the number of congruences in a quotient lattice (mathematically $\log \left(\left|L^{t} / \theta(J)\right|\right)$. The number of congruences is the number of partitions in a lattice. For example, the number of congruences is two for Figure 1b (ii). A zero bit flip rate occurs only when the number of congruence is one in Figure 2d. The correlation coefficient in Figure $2 \mathrm{~d}$ is 0.68 . The correlation relationship between these two factors suggests that the bit flip is not entirely random. The structure of the evolving lattice affects the value of bit flip rate. The bit flip rate temporally changes without any parameter tuning.

Finally, we point out that the update algorithm of our model is not synchronous. Figure 2e shows the proportion of unchanged individuals with time. This graph suggests that whole population always contains some individuals who keep their state for the updating algorithms. In other words, some individual may change with time and other individuals may not change with time. The discrete time updating in our model is essentially asynchronous in this sense. This asynchronous (or heterogeneous) update comes from the congruence of the lattice for a given reference point. Precisely, unchanged individuals correspond to their fitness belonging to the congruence of the top element (or mathematically individual whose the fitness in $\left.[11 \ldots 1]_{\theta(J)}\right)$. From the definition of the multiplicity of fitness, we can introduce this asynchronous update rule.

Estimating the lifespan distribution is very important when considering evolutionary or ecological systems [15]. The power law with a slope of -2 is universally observed in many biological events, such as the fossil record $[16,17,18]$. Figure 3 shows that our model also has a -2 power law for the lifespan distribution of 7-bit, 8-bit and 9-bit strings over 100,000 simulated steps. In this study the lifespan is the length of time steps with one kind of $n$-bit string exist in the population. Bit strings that are far from the target will be more likely to become extinct. By contrast, bit strings that are near by the target would have a high probability of survival. Power law distribution means that there is no characteristic scale for extinction events of a species. The power law suggests that species never behave independently but rather interact with each other through incomplete information. In our model, we construct a lattice from disjointed fitness blocks. The bit flip rate, which determines extinction events, is regulated by an evolving lattice. This regulation of our model inserts the global information to the population dynamics.

Evolving lattices also affect oscillations for the average distance from the target. Figure 4 shows a power spectrum for Figure 2b. We define $f$ as the frequency and $S(f)$ as the power spectrum. This generates the mathematical expression $S(f) \sim f^{-\gamma}$. The slope of the graph is $\gamma=-1.36 \pm 0.02$. In other words, Figure 4a shows a $1 / f$ fluctuation. This relationship indicates that adapting the target bit string at different times correlates over a very long time scale. Here we discuss the importance of a reference point for $1 / f$ fluctuations. We examined what would happen to the power spectrum without a reference point. We defined a control system in which the reference point never contains any global information. In other words, an element that leads to an ideal is randomly selected from an evolving lattice without a reference point. If the 
model lacks a reference point, the distribution of the power spectrum resembles Figure 4b. The graph becomes flat for high frequencies. We examined both slopes in a high-frequency region $(0,0.05]$ for Figure 4a and Figure 4b. We obtained slopes of $-1.08 \pm 0.02$ (Figure $4 \mathrm{a}$ ) and $-0.45 \pm 0.05$ (Figure $4 \mathrm{~b}$ ). The difference between the slopes is more than double. Figure 4b contains two essential suggestions of our model. The slope of the low frequency regions directly influences the evolving lattice structures. Adaptive behavior on a long-range time scale is performed by the global lattice structures. The other component is that two divided regions in a power spectrum suggest that $1 / f$ fluctuations result from the collaboration between the evolving lattice structure and the method used to select its reference point. By adding a reference point in a lattice, a species can adjust on shorter time scales.

Next, we compared our evolving lattice model to the control model, which we called a " $\mu-\rho$ model”. The details of the algorithm are described in the Appendix. Here, we present the main concept of the $\mu$ - $\rho$ model. The $\mu$ - $\rho$ model shares a common feature in fitness blocks. Each species ( $n$-bit string) produces a fitness block that is coupled with the target and is articulated into two regions that contain unchanging and changing digits. The evolving lattice model changes fitness blocks using a quotient lattice, but the $\mu$ - $\rho$ model changes fitness blocks with a particular probability distribution, which we call the mutation rate, $\mu$ (this concept and the word relies on Eigen's model [9]) and the overestimation rate, $\rho$. The mutation rate $\mu$ indicates the probability of a copying error. All fitness blocks are maintained if the mutation rate is zero. However, if the mutation rate becomes larger, unchanging digits can be replaced with changing digits. When the mutation rate is high, the fitness of the species becomes low and the bit flip rate becomes high. By contrast, the overestimation rate $\rho$ has an opposite role. By tuning $\rho$ we can control if the changing digits in the fitness block would be unchanged with a probability $\rho$. Therefore, when $\rho$ is high, each species overestimates its own fitness. Tuning these parameters $(\mu$ and $\rho$ ) causes the model to have some corrective abilities. To compare our model to the probabilistic model, we discuss the essential differences in the adaptation process between the evolving lattice model and the probabilistic model.

Figure $5 \mathrm{a}, 5 \mathrm{~b}, 5 \mathrm{c}$ and $5 \mathrm{~d}$ show the behavior of the $\mu-\rho$ model when the mutation rate and the overestimation rate are controlled. Figure $5 \mathrm{a}(5 \mathrm{~b})$ is the relationship between the two parameters and the mean (standard variation) of the average distance from the target bit strings. The mean and the standard variation of the average distance strongly depend on the mutation rate $\mu$ in both figures. The mean averaged distance is low (or the fitness is high) if the mutation rate is low and vice versa. We observed that the standard deviation of the average distance also becomes larger when the mutation rate is high. Figure 5a suggests that the overestimation of the fitness generally prevents raising the fitness. Figure $5 \mathrm{c}(5 \mathrm{~d})$ shows the relationship between the two parameters and the mean (standard variation) of the bit flip rate. The mutation rate $\mu$ also influences to the bit flip behavior. Additionally, the overestimation rate decreases the mean and the standard variation of the bit flip rate. By setting these two parameters to be low, the species in the $\mu-\rho$ model show a -2 power law for the lifespan (for example, see Figure 5e).

Especially, we find that Figure 5a and 5b provide interesting information for our evolving lattice model. In our evolving lattice model, each fitness block tends to be overestimated from its original fitness value by 
using a quotient lattice. So far, we observed that the overestimation of the fitness plays an important role for the adaptive behavior to the target bit string. However, the overestimation parameter $\rho$ never can do a little contribution to the adaptive behavior (Figure 5a and 5b). From this fact, we can conclude that the overestimation of the fitness never works out appropriately when the overestimation of fitness is determined stochastically by tuning the parameter $\rho$. The context-dependent properties through the congruences in a lattice and the reference point give species a proper usage of the overestimation of the fitness to approach the target bit string.

The power law for the lifespan is observed when both parameter values are low. Therefore, we fixed the parameters at $\mu=\rho=0.005$ for comparison to the evolving lattice model. We ran the simulation using 8-bit strings. First, we compared the average bit flip rate for both models. Figure $5 f$ shows the result of this comparison. The black bar corresponds to the evolving lattice model, and the gray bar corresponds to the $\mu-\rho$ model. The graph shows that the average bit flip rate of the $\mu-\rho$ model is three times greater than the evolving lattice model. In other words, the average bit flip rate of our model is very small (this value is smaller than the $\mu$ - $\rho$ model when $\mu=\rho=0$ ). We point out that bit flip rate of the $\mu$ - $\rho$ model rarely becomes zero as was seen in the evolving lattice model in Figure 2c because the error of the fitness block is determined stochastically. If the population is larger, it becomes impossible for no bit flips to occur in every species. Unlike the $\mu$ - $\rho$ model, the bit flip rate of the evolving lattice model never solely depends on the probability but also depends on a quotient lattice structure (see Figure 2d).

Figure $5 \mathrm{~g}$ also provides interesting information. We examined the mean (standard deviation) of the variance around the average distance between the target and the species. The colors of Figure $5 \mathrm{~g}$ are the same as in Figure $5 f$. The mean variation of the average distance in the $\mu$ - $\rho$ model is eight times lower than the evolving lattice model. This indicates that the distance to the target rarely fluctuates around the mean average distance. High bit flip rates do not lead the $\mu$ - $\rho$ model to high fitness; instead the model maintains low fitness. However, the evolving lattice model fluctuates widely around the mean average distance despite low bit flip rates. This suggests that the evolving lattice model efficiently uses its bit flips to approach the target bit string. A highly efficient use of bit flipping has never been observed for the probabilistic $\mu$ - $\rho$ model because the mutation rate is uniformly distributed among bit strings.

\section{Discussion}

The main achievements in our study are roughly divided into three parts. One is that our evolving lattice model satisfies SOC (self-organized criticality) properties such as -2 power law for the lifespan distribution and $1 / f$ fluctuation in adapting process. Second, the importance of the reference point, which corresponds the historical property of the fitness blocks, supports whole species adapting behavior as one organism (emergence of $1 / f$ fluctuation). Third, the overestimation of the fitness, induced by a quotient lattice, may have a possibility of the model of exaptation by not using stochastic way.

First, we observed that the evolving lattice model has properties such as power laws for the lifespan 
distribution and $1 / f$ fluctuations for the adaptive process. Our model never needed external bit flip events but instead generates inherent bit flip events by negotiating between the local fitness and global context (quotient lattice). The word inherent here means that the rate of bit flips is never controlled by external parameters. The flipping rate of each bit string is determined by the structure of a lattice at time $t$. The importance of the inherent bit flip rate becomes clear when compared to the $\mu$ - $\rho$ model. The $\mu$ - $\rho$ model controls the bit flip rate with the parameter $\mu$. In other words, the bit flip events in each bit string are determined stochastically. We observed that this stochastic event never showed variability in adapting to the target. We also observed that the bit flip rate of the evolving lattice model was much smaller than that of the $\mu$ - $\rho$ model and that our model showed high variability. This suggests that inherent bit flip events are used efficiently to approach the target bit string. Species in the evolving lattice model use fewer noise-like events to adapt to the environment (the target bit string).

Next we considered the role of the reference point in our model. We suggested the importance of the reference point in the lattice structure in the adaptation process. The reference point becomes the criteria when we make an ideal. This ideal induces a quotient lattice. With a quotient lattice, all of the fitness blocks change their element to the upper limit of the congruence. Therefore, lower order elements are substituted with upper elements in the lattice. We observed that this overestimation of the fitness block through the quotient lattice is important for the population dynamics of the species. However, we also observed that it was insufficient for the emergence of $1 / f$ fluctuation if there was no reference point. There was a lack of intensity over short time scales. The reference point in the evolving lattice served to adjust the high frequency. Recall that the reference point was selected from set $\bar{B}$. This selection suggests that a selected reference point has a property of an element as local information (one of the fitness blocks) and a set of global information (a quotient lattice). In other words, the double purpose of the reference point in a lattice activates more dynamic negotiations between global and local information for adaptive processes.

Here we comment on the possibility of modeling the exaptation (or preadaptation) in adaptive process. The definition of the exaptation was the multiplicity of fitness in nature. We can consider the multiplicity of fitness as "species dares to keep its traits which seem unnecessary" because the fitness usually is defined on one dimension (in other words, species has only one criterion for measuring its fitness). The multiplicity of the fitness was represented as the overestimation of the fitness in our evolving lattice model. Furthermore, the overestimation of the fitness was not determined by using stochastic ways but by using a reference point, which represented a historical property. This historical dependency of the fitness means that the local fitness estimation of each species is not separable from their global context. Therefore, the exaptation, in our model, is interpreted as a result of the negotiation between local fitness and global context.

Finally, we emphasize the important difference between our model and self-organized criticality (SOC). We showed that our model shows the power law and 1/f fluctuation that are often observed in SOC [18, 19, 20, 21]. The concept of SOC is that nature evolves toward the critical state without any fine-tuning. Each species in the critical state connects to each other species for various times and on special scales. The power law and $1 / f$ fluctuations suggest self-similar structures for biological events. Bak et al. suggests that each species constructs one organization when the systems are in the critical state [3, 4]. Recently, it found that 
flocks could move as one corrective unit because the collection of birds is at the critical state [22]. Researchers often discuss the relationship between SOC and these emergent behaviors as an organism. In fact, there are many studies that insist that biological events (fossil record, corrective behavior and ecology $[15,16,17])$ can be explained by SOC. However, the emergence of SOC remains a controversial issue.

The most general explanation of the emergence SOC is the balance between two factors, such as the balance between order and disorder or the balance between birth and death, etc. The simplest example is the random walk model, which is discussed by Yang [23]. He considered a moving particle on a one-dimensional line. This particle can move right or left stochastically. In this model, the lifespan is the time taken to return to the starting position. A power law of the lifespan distribution emerges when the probability of moving in a direction equals $1 / 2$. The particle reaches the critical state when we assign equal probability. Here we must pay attention to the globality that invades into this interpretation. Considering the balance between two powers is possible only when the state of the two extremes is static. There is no room for considering the negotiation between local and global information once we consider the balance between the two extremes. This static view emerges as the threshold, least fit species and an equal probability of changing direction [3, $4,19,20,21,23]$. The next question that emerges is the origin of the balance between two factors.

To consider the negotiation between global condition and local fitness, we must avoid a static assumption between the two factors. It would be difficult to explain the origin of the criticality if we assume these static perspectives. Our evolving lattice model proposes a different perspective. Both the relation between the global and local fitness of our model change dynamically. The dynamic local fitness emerges as incomplete information for an environment. The dynamic global information emerges as a quotient lattice global by using a reference point at every step. The incomplete information of the fitness never works negatively here but instead drives the active negotiation between globality and locality. There is no perspective for finding the balance between them in our model. Our method will become important when we grasp the emergent properties of biological phenomena.

\section{Appendix}

We give an explanation for a comparative model. The concept of the model comes from an ideal target and its error. Each species tries to adapt to its environment, which is represented by the target bit string. The target also has a randomly determined hidden digit for every step. Then algorithm is as follows.

(1) Generate $m n$-bit strings $\left\langle s^{1}\right\rangle_{n}^{0},\left\langle s^{2}\right\rangle_{n}^{0}, \ldots\left\langle s^{m}\right\rangle_{n}^{0}$ and a target $n$-bit string $\langle t\rangle_{n}$ randomly.

(2) Randomly select a hidden digit of the target from $\{1,2, \ldots, n\}$. Make fitness blocks from a set of $n$-bit strings $M^{t}=\left\{\left\langle s^{1}\right\rangle_{n}^{t},\left\langle s^{2}\right\rangle_{n}^{t}, \ldots,\left\langle s^{m}\right\rangle_{n}{ }^{t}\right\}$ using Definition 3.2. A set of binary bit strings of fitness blocks is denoted by $B^{t}=\left\{\left\langle b^{1}\right\rangle_{n}^{t},\left\langle b^{2}\right\rangle_{n}^{t}, \ldots,\left\langle b^{l}\right\rangle_{n}^{t}\right\}$. 
(3) Change each $n$-bit string using fitness blocks. For $1 \leq j \leq n$ and $1 \leq i \leq m$,

$$
s_{j}^{i, t+1}=\left\{\begin{array}{c}
\left\{\begin{array}{c}
s_{j}^{i, t} \text { if } b_{j}^{i, t}=1 \text { and probability }(1-\mu) \\
0 \text { or } 1 \text { if } b_{j}^{i, t}=1 \text { and probability } \mu
\end{array}\right. \\
\left\{\begin{array}{c}
s_{j}^{i, t} \text { if } b_{j}^{i, t}=0 \text { and probability } \rho \\
0 \text { or } 1 \text { if } b_{j}^{i, t}=0 \text { and probability }(1-\rho)
\end{array}\right.
\end{array}\right.
$$

and construct a new set for species $M^{t+1}=\left\{\left\langle s^{1}\right\rangle_{n}{ }^{t+1},\left\langle s^{2}\right\rangle_{n}{ }^{t+1}, \ldots,\left\langle s^{m}\right\rangle_{n}{ }^{t+1}\right\}$.

Repeat steps (2) - (3). $\mu$ is a parameter representing the mutation rate for each bit in a string. $\rho$ is a parameter for the overestimation of the fitness. Each species can mimic completely without a hidden digit when $\mu$ and $\rho$ equals zero. By tuning these two parameters, we can obtain the critical behavior such as -2 power law.

\section{Reference}

1. Adami. C. Self-Organized Criticality in Living Systems. Phys. Lett. A. 203 (1995) 29-32.

2. Bak, P. and Sneppen, K., Punctuated Equilibrium and Criticality in a Simple Model of Evolution. Phs. Rev. Lett. 71 (1993), pp. 4083-4086.

3. Bak, P., Tang, C. and Wiesenfeld, K. Self-Organized Criticality: An Explanation of 1/f Noise. Phys. Rev. Lett. 59. (1987) pp. 381- 384.

4. Buss, D.M., Haselton, M.G., Shackelford, T.K., Bleske, A.L. and Wakefield, J. C. Adaptation, Exaptations, and Spandrels. American Psychologist. 53. (1998). pp. 533-548.

5. Cavagna, A., Cimarelli,C., Giardina, I., Parisi,G., Santagati, R., Stefanini, F. \& Viale, M. Scale-Free Correlation in the bird flocks. Proc. Natl. Acad. Sci. USA. (2010).

6. Davey, B. A. and Priestelely, H. A. Introduction to Lattices and Oder. Cambridge. Cambridge University Press, 2005.

7. Darwin, C. The Origin of Species by Means of Natural Selection, 1859.

8. Eigen M, McCaskill J, Schuster P: The Molecular Quasi-Species. Adv Chem Phys 75 (1989) pp.149-263.

9. Gould, S.J. Exaptation: A Crucial Tool for Evolutionary Psychology. Journal of Social Issues, 47 (1999) pp. 43-65.

10. Gunji, Y-P., Haruna, T. and Sawa, K. Principles of Biological Organization: Local-Global Negotiation Based on “Material Cause”. Physica D. 219 (2006) pp. 152-167.

11. Jensen, H. J. Self-Organized Criticality: Emergent Complex Behavior in Physical and Biological Systems. Cambridge. Cambridge University Press, 1998.

12. Jansen, V. A. A. and Stumpf. M. P. H. Making Sense of Evolution in an Uncertain World. Science. 309 (2005) pp. 2005-2007.

13. Lande. R. and Shannon. S. The role of Genetic Variation in Adaptation and Population Persistence in a Changing Environment. Evolution. 50. 1996. pp. 434-437. 
14. MacColl, A. D. C., The Ecological Causes of Evolution. Trends in Ecology and Evolution. 26 (2011), pp. 514-522.

15. Mustonen, V. and Lässig, M. Fitness flux and Ubiquity of Adaptive Evolution. Proc. Natl. Acad. Sci. USA. 107 (2010) pp. 4248- 4253.

16. Newman, M. E. J. and Eble, G. J. Power Spectra of Extinction in the Fossil Record. Proc. R. Soc. Lond. B. 266 (1999) pp. 1267-1270.

17. Newman, M. E. J. and Eble, G. J. Decline in Extinction Rates and Scale Invariance in the Fossil Record. Paleobiology 2 (1999) pp. 434-439.

18. Pigolotti, S. Flammini, A. Marsili, M. and Maritan, A. Species Lifetime Distribution for Simple Models of Ecologies. Proc. Natl. Acad. Sci. USA. 102 (2005) pp. 15747- 15751.

19. Sneppen, K., Bak, P, Flyvbjerg, H. and Jensen, M. H. Evolution as a Self-Organized Critical Phenomenon. Proc. Natl. Acad. Sci. USA. 92 (1995) pp. 5209- 5213.

20. Vecchio, D. D., Murray, R. M. and Klavins, E. Descrete State Estimators for Systems on a Lattice. Automatica. 42 (2006) 271-285.

21. West, G. B. and Brown, J. H. Life’s Universal Scaling Laws. Physics Today. (2004) pp. 36- 42.

22. Wilke, C. O. Quasispecies Theory in the Context of Population Genetics. BMC Evolutional Biology. 5 (2005).

23. Yang. C. B. The origin of Power-law Distribution in Self-Organized Criticality. J. Phys. A: Math. Gen. 37. (2004) L523-L529. 
Figure 1

(a)
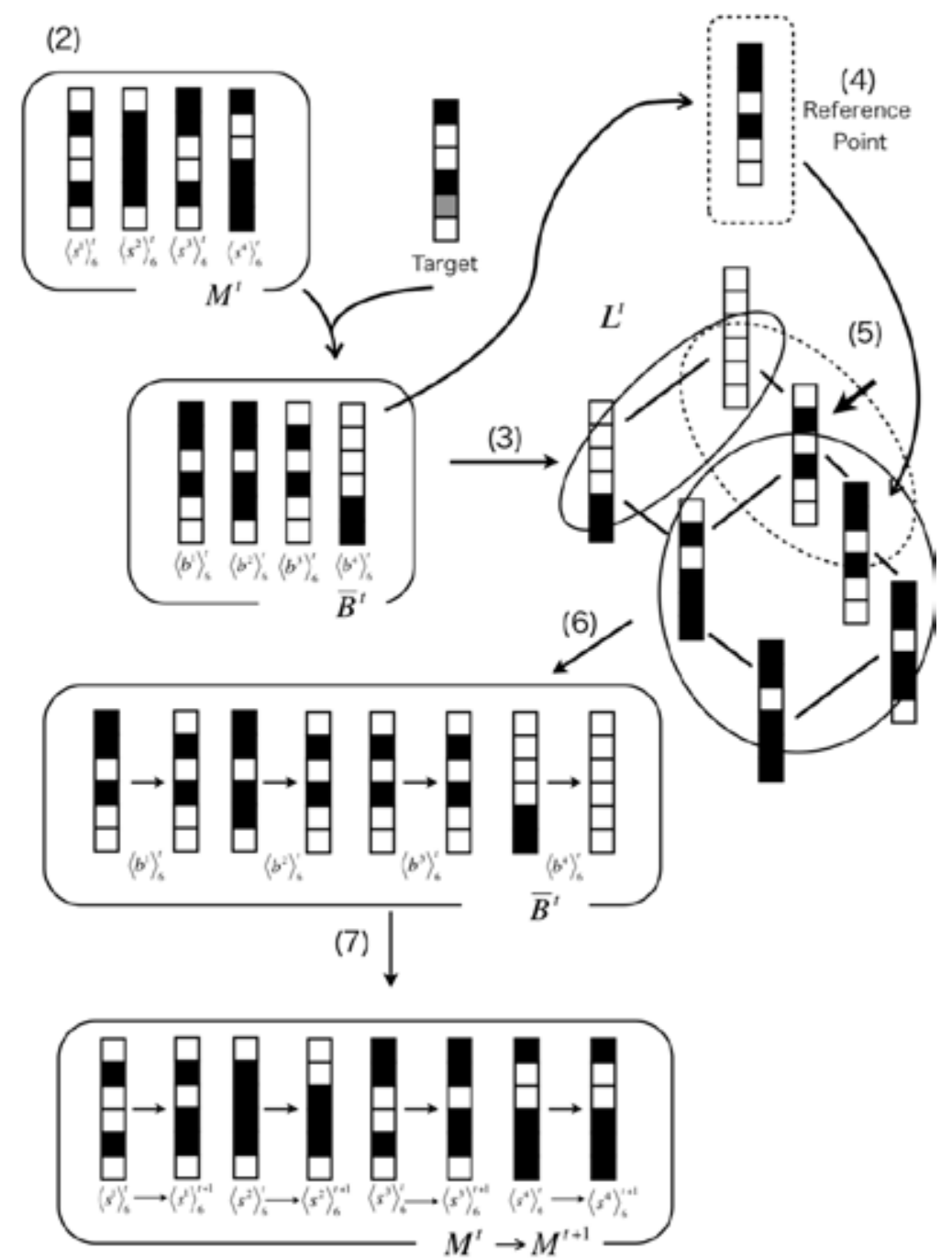

(b)

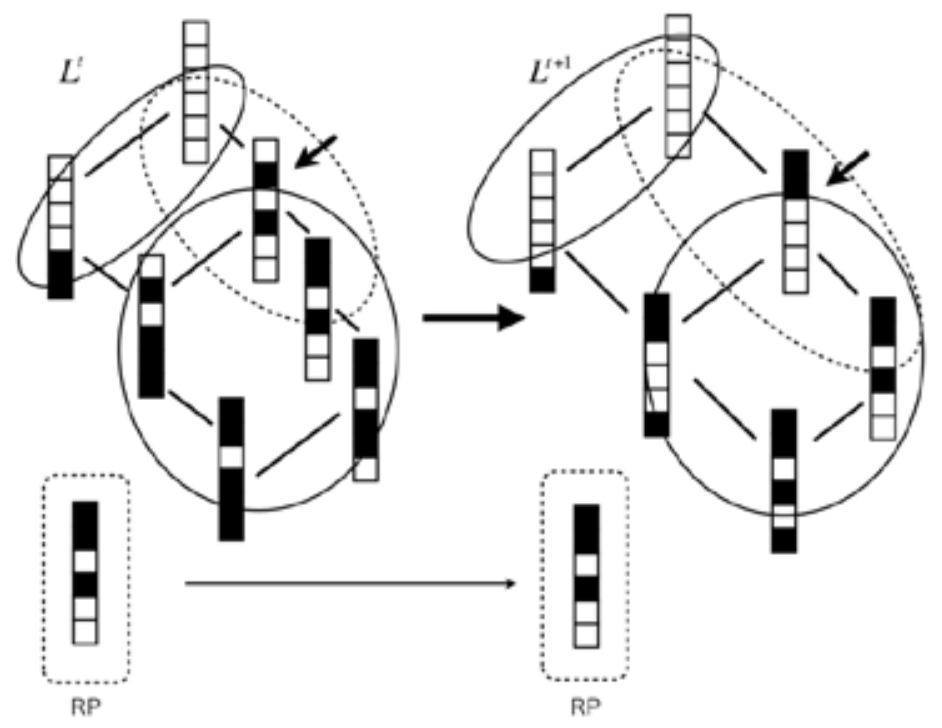


(c)

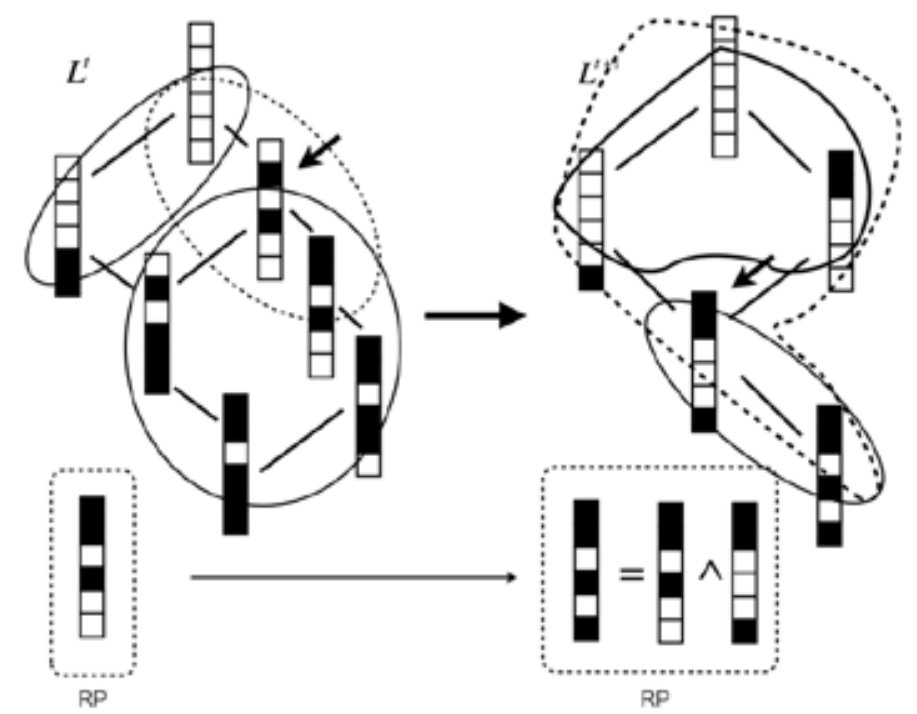

(a) Algorithm for one step of the evolving lattice model. We consider the case that four species try to mimic the target. We have colored each bit of a bit string black (zero) or white (one). Gray areas in the target bit string represent the hidden digit at time $t$. The number in the figure is the index of the algorithm in the main text. In (4), the reference point is selected from a set of fitness blocks. The thick arrow in (5) indicates a selected top element for the ideal. The dotted circle in (5) is the filter for the reference point. The solid black circles indicate congruence in lattice $L^{t}$. The thin horizontal arrows in (6) and (7) indicate the transformation of bit strings for fitness blocks and species.

(b) A case where the reference point (RP) exists in the next evolved lattice, $L^{t+1}$. The thick arrow indicates the selected top element for the ideal. We use the same RP as in Rule 2.

(c) A case where the reference point (RP) does not exist in the next evolved lattice, $L^{t+1}$. However, in this case, the intersection between the RP in $L^{t}$ and the element in $L^{t+1}$ exists in the lattice. We choose the RP using Rule 2. The thick arrow indicates a selected top element for the ideal. For the other cases, the $\mathrm{RP}$ is selected from a set of fitness blocks as in Figure 1 (a).

\section{Figure 2}

(a)
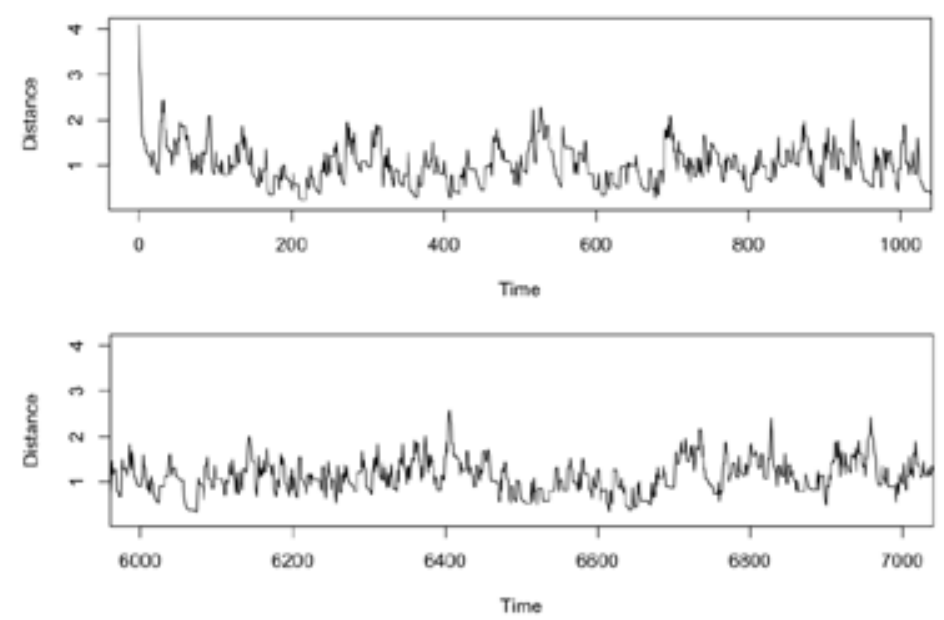
(b)
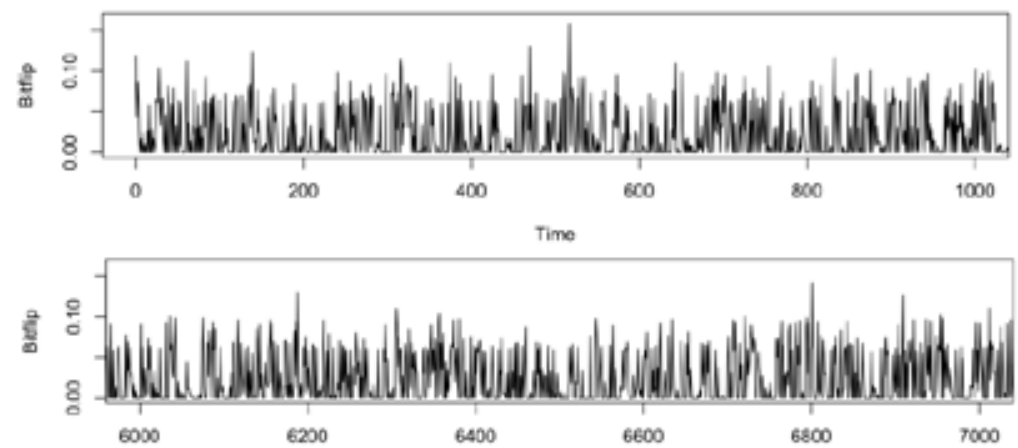

(c)
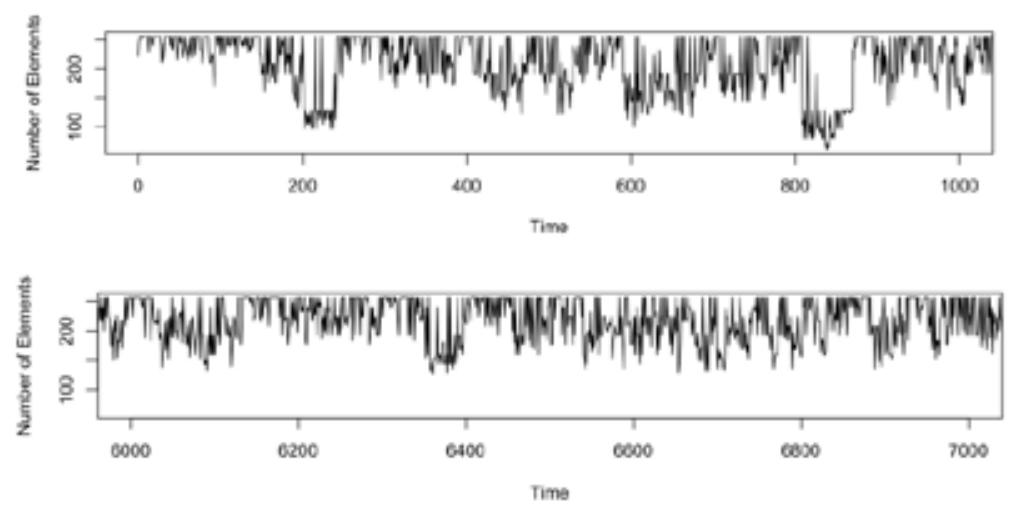

(d)

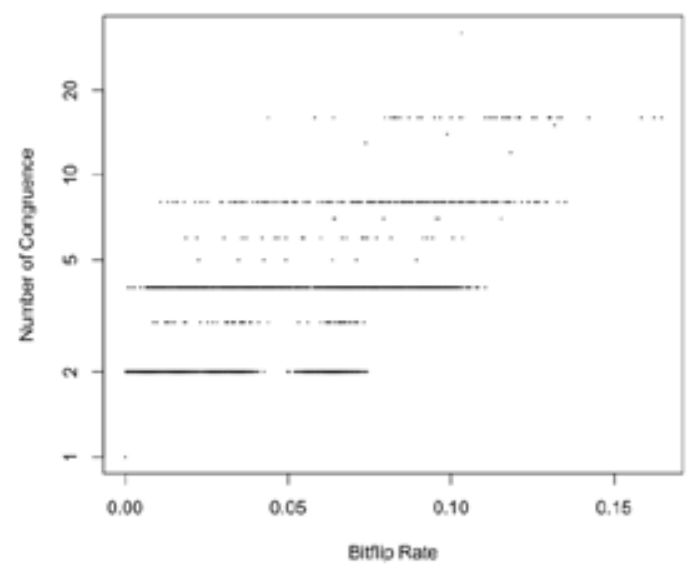

(e)

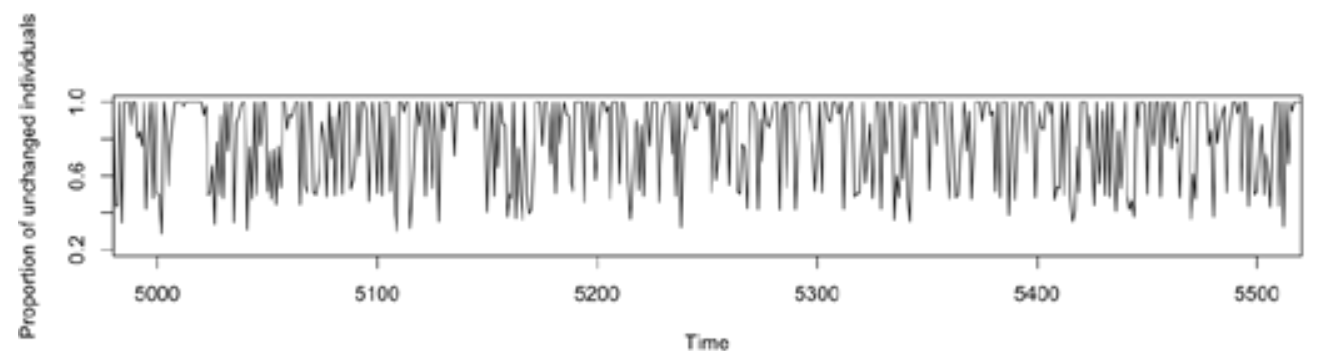


(a) The graph shows a time series for the number of elements in a lattice. The constructed lattice is never static but always behaves dynamically.

(b) The graph shows a time series for the average distance between a target and a species. Lower distances indicate higher fitness for the species. We observe an oscillation in this graph. The mean average value is approximately 1.1 .

(c) The graph shows a time series for the average bit flip rate per bit. The bit flip rate is determined by fitness blocks and a quotient lattice at each step.

(d) The relationship between the bit flip rate and the log transformed number of congruences is shown in the quotient lattice, which is constructed from fitness blocks. There is a roughly correlative relationship between them. The correlation coefficient is 0.68 .

(e) The time series of the proportion of unchanged individuals in whole population. The rate of unchanged individuals changes dynamically with time. This graph suggests that the update of each state is not synchronous.

\section{Figure 3}
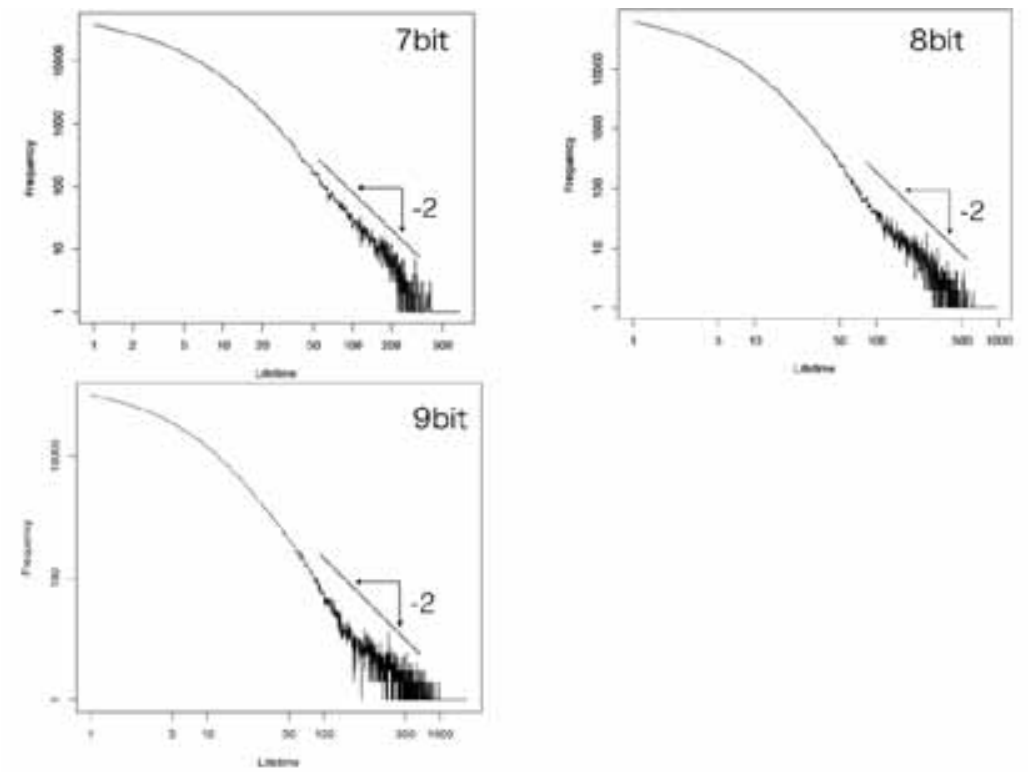

The distribution of the lifespan of each species for 8-bit and 9-bit strings. The vertical line is the frequency and the horizontal line is the lifespan of each species. We plotted them as a log-log plot. Both bit strings show the power law. The slopes of the graphs are $-2.17 \pm 0.15$ (7-bit strings), $-2.18 \pm 0.07$ (8-bit strings) and $-2.04 \pm 0.03$ (9-bit), respectively. We simulated 100,000 steps in each case. 


\section{Figure 4}
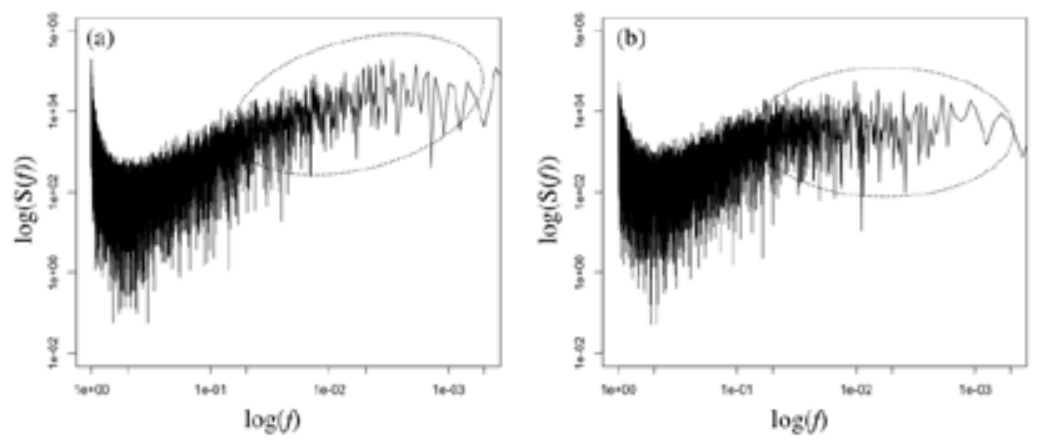

A power spectrum $S(f)$ for the oscillations in Fig. 2a. The slope in Figure 2a is $-1.36 \pm 0.02$. The graph shows $1 / f$ noise for the species' adaptive behavior. Figure $4 \mathrm{~b}$ is a power spectrum $S(f)$ for the oscillation in Fig. 2a without a reference point. The graph becomes flat at high frequencies. Species without a reference point do not show a high intensity over short time scales. This suggests that the low intensity of long time scales is solely a result of global constraints, which correspond to a series of lattice structures. The slopes in the dotted region are $-1.08 \pm 0.02$ (Figure $4 \mathrm{a}$ ) and $-0.45 \pm 0.05$ (Figure $4 \mathrm{~b}$ ), respectively.

\section{Figure 5}

(a)

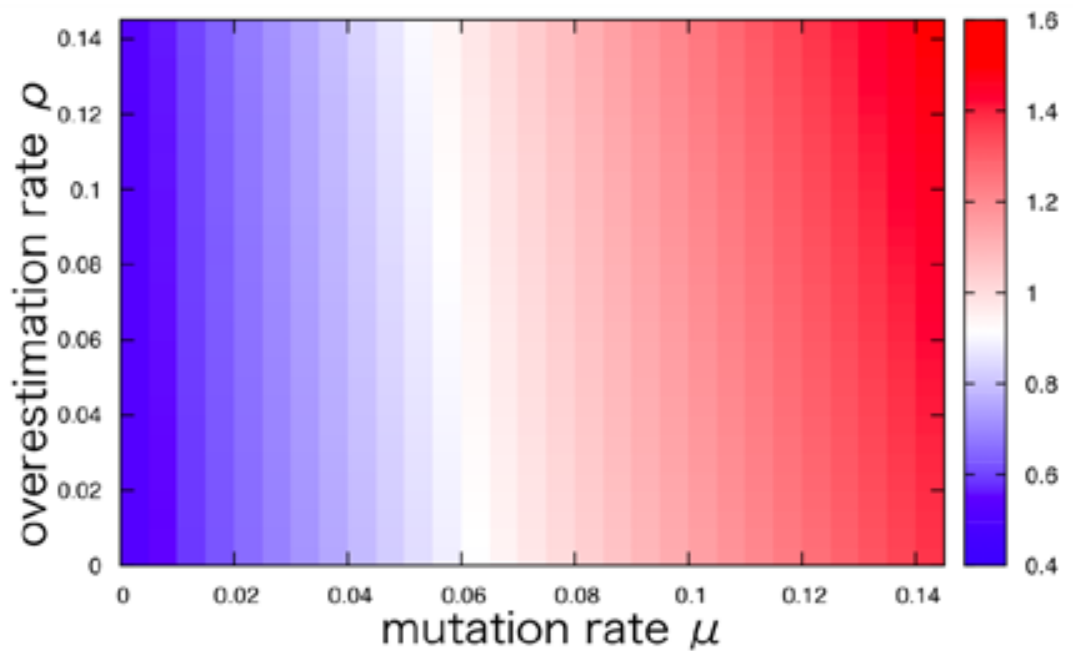

(b)

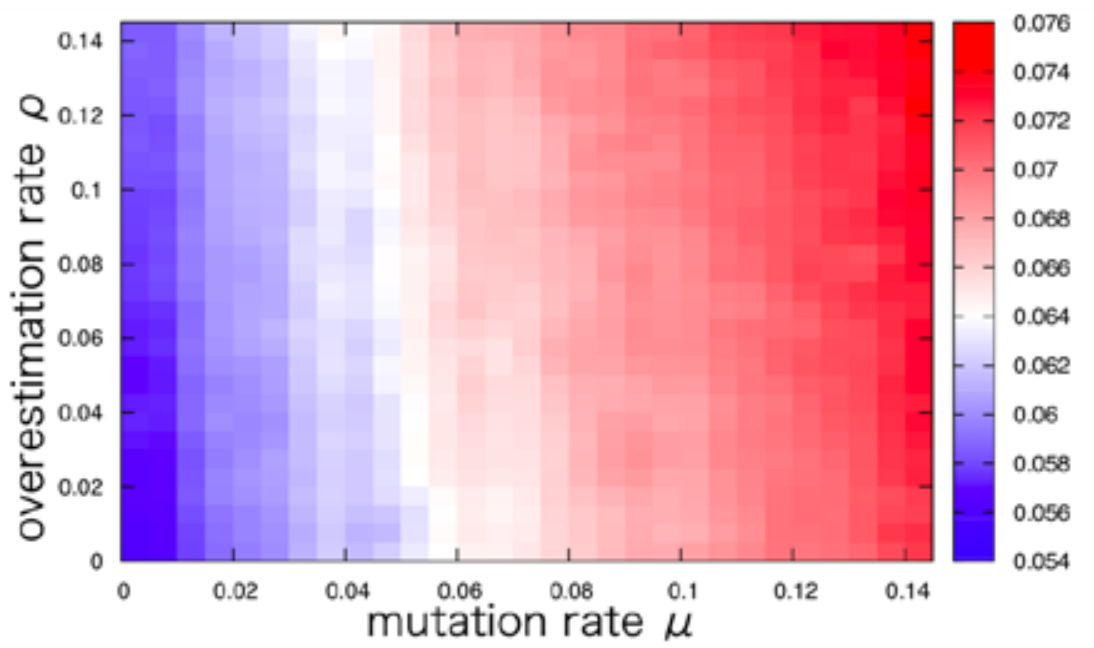


(c)

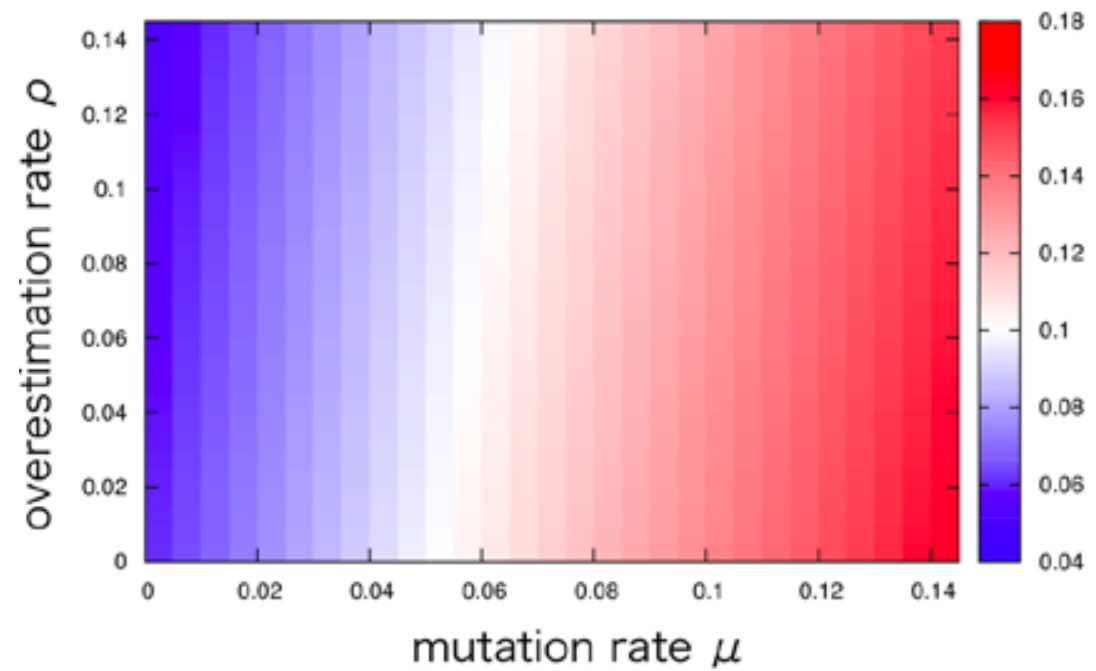

(d)

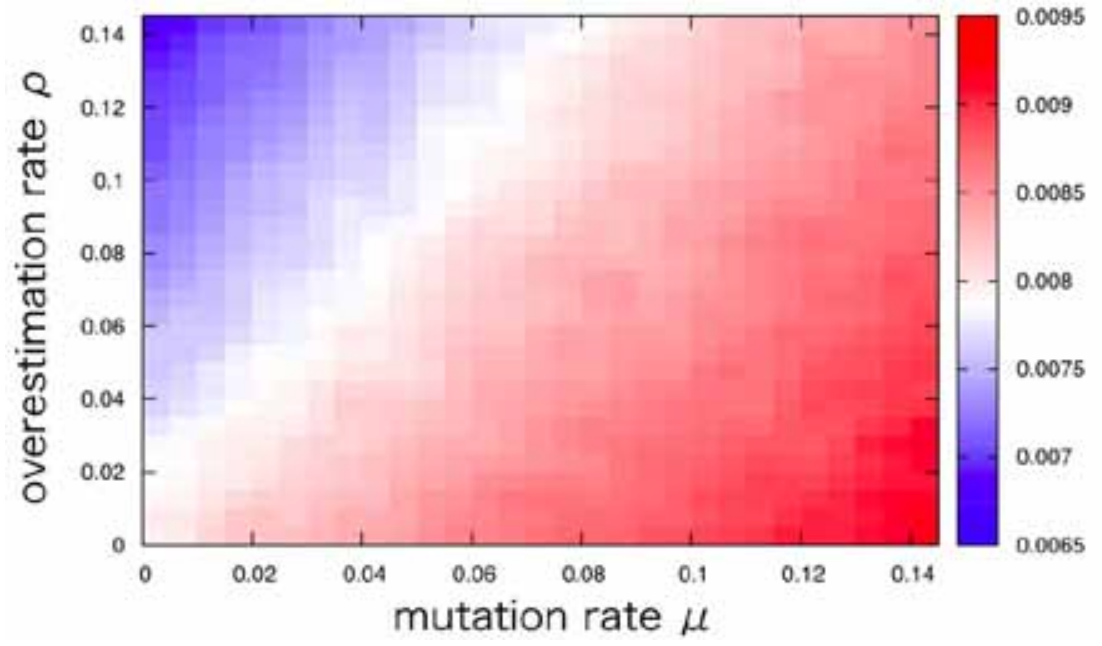

(e)

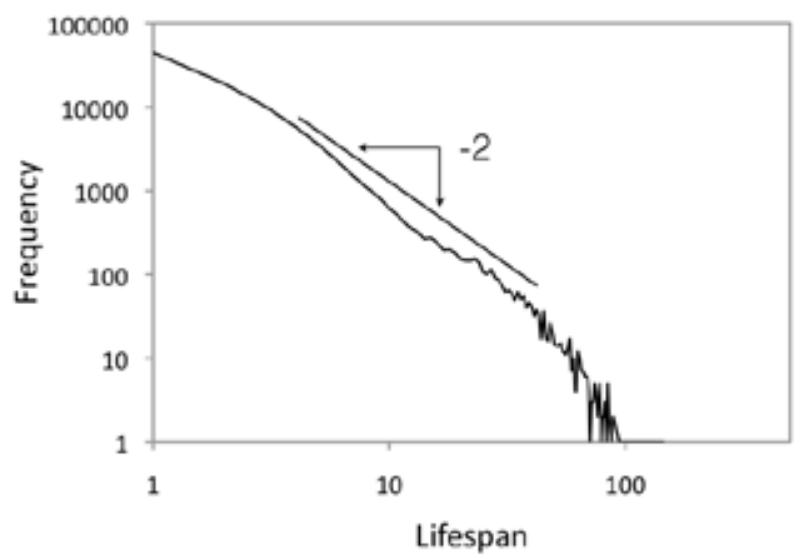


(f)

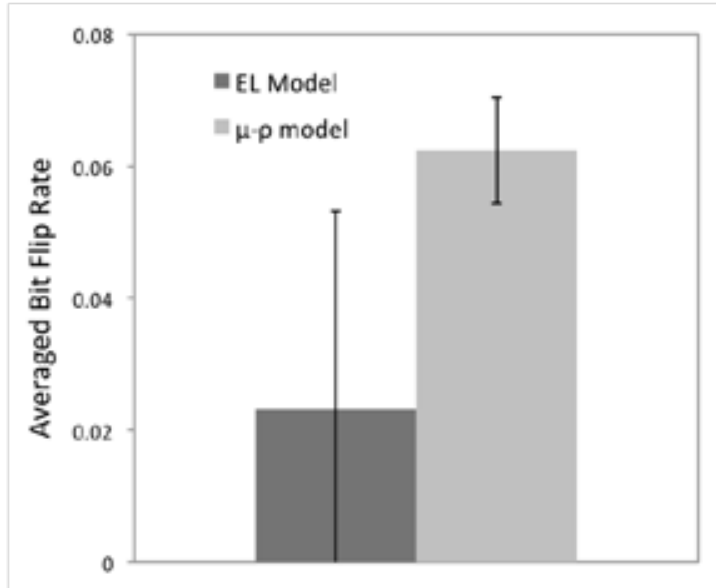

(g)

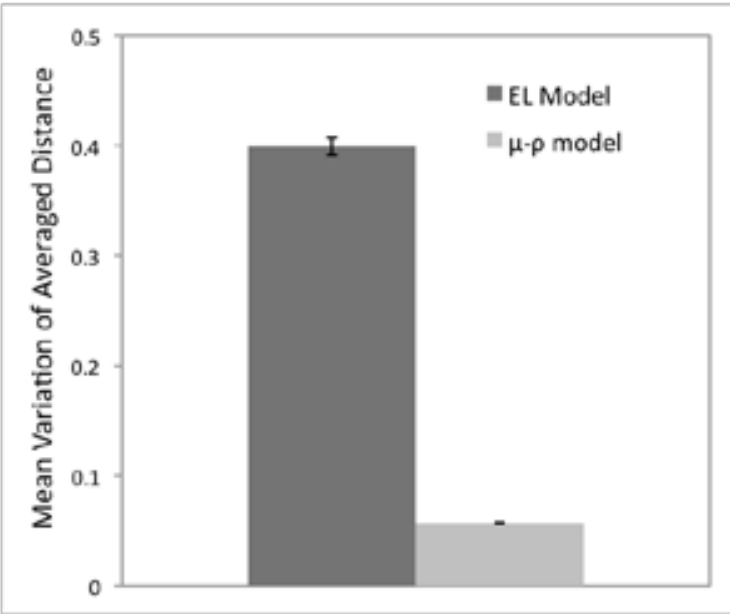

Figure 5a, 5b, 5c, $5 d$ and 5e are graphs for the $\mu$ - $\rho$ model (8-bit string). The details of the algorithm are listed in the Appendix.

(a) The mean average distance from the target as a function of the mutation rate, $\mu$, and an overestimation rate, $\rho$. The average distance from the target is highly dependent on $\mu$. The influence of $\rho$ is relatively small compared to $\mu$.

(b) The standard deviation of the average distance from the target as function of the mutation rate, $\mu$, and the overestimation rate, $\rho$. It shows the same tendencies as Figure 5a.

(c) The mean bit flip rate as function of the mutation rate, $\mu$, and the overestimation rate, $\rho$. The mean bit flip rate also depends on $\mu$. The overestimation plays a role in decreasing the mean bit flip rate.

(d) The standard deviation of the bit flip rate as function of the mutation rate, $\mu$, and the overestimation rate, $\rho$. In contrast with other three figures, the standard deviation is highly dependent on $\rho$.

(e) Example of the power law relationship between life span and frequency. This relationship can be obtained under low values for both the mutation rate and the overestimation rate. In this case, we set the two parameters to $\rho=0.005$ and $\mu=0.005$. The slope of the line is roughly -2 .

The next two graphs show a comparison between the evolving lattice model and the $\mu$ - $\rho$ model. The parameters $\mu$ and $\rho$ are fixed at $\mu=\rho=0.005$. The number of species is unified at $2^{8}=256$. 
(f) Both bars show the average bit flip rate for the evolving lattice (black) and the mutation (gray) models. The error bars represent the standard deviation. The value of the average bit flip rate for the evolving lattice model is three times lower than the $\mu$ - $\rho$ model.

(g) Both bars show the mean variation from the average distance for the evolving lattice (black) and the mutation (gray) models. The error bars represent the standard deviation. The value for the evolving lattice model is eight times greater than that of the $\mu-\rho$ model. 